\title{
Knowledge, Attitudes and Practices Regarding Traditional and Complimentary Medicine in Oman
}

Hassan Al-Riyami, ${ }^{1}$ Ahmed Al-Maskari, ${ }^{1}$ "Sunil K. Nadar, ${ }^{2}$ Mohammed Almaskari ${ }^{3}$

ABSTRACT: Objectives: This study aimed to assess the knowledge, attitudes and practices regarding traditional medicine (TM) in Oman and examine the factors that necessitate its use. Methods: This cross-sectional, questionnairebased study was conducted among the general population from November 2019 and March 2020. All Omani nationals above the age of 18 were eligible to be enrolled. The questionnaire consisted of questions on the knowledge, attitudes and use regarding traditional medicine in Oman. Results: A total of 598 responses to the questionnaire were received (response rate: $85.4 \%$ ), of which 552 were deemed complete. Most responses were received from males (62.5\%) and the sample had a mean age of $33.6 \pm 7.7$ years. A majority of the respondents $(90 \%)$ were aware of the different types of TM in Oman; a high percentage (81.5\%) felt that it was effective. Most (67.8\%) had tried at least one method of TM use. Individuals who were older had tried TM compared to those who had not ( $34.5 \pm 7.8$ years versus $31.8 \pm 7.2$ years; $P<0.001)$; in addition, more males than females $(72.2 \%$ versus $27.8 \% ; P<0.001)$ and those with full-time employment than those without had tried TM $(84.2 \%$ versus $14.2 \% ; P<0.001)$. Herbal medications $(65.8 \%)$ and traditional massage (60.4\%) were the most common forms of TM practice. Among females, herbal medications (69.2\%) and massage (63.5\%) are most often used; among males, cupping (65.2\%) followed by herbal medications (64.4\%) and massage (59.3\%) were used more often. Notably, back pain (74.3\%) was the most common condition for which TM was reportedly used, with only a small percentage (8.3\%) reporting any concomitant side-effects. Conclusion: There is widespread use of TM among Oman's urban population. An improved understanding of their benefits will facilitate their incorporation into modern health care services.

Keywords: Traditional Medicine; Complementary Medicine; Knowledge; Attitude; Oman.

\section{Advances IN KNOWLEDGE}

The study demonstrates that traditional medicine is still widely practiced in Oman.

This study is the first in the region to evaluate the knowledge, attitudes and practices of traditional medicine among the urban population in Oman.

\section{Applications to Patient Care}

Understanding the use of traditional medicinal practices will help physicians evaluate their patients better and obtain better medical histories from them upon presentation, revealing any side-effects or complications related to these traditional medicinal practices.

Such an understanding will also aid the formulation of healthcare plans at a governmental level, as experts will be able to streamline the integration of these traditional practices with modern healthcare.

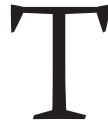
Raditional medicine (TM) is a term broadly used to refer to various forms of indigenous medicine practiced by communities native to a particular region, such as the traditional Chinese medicine system, the Indian Ayurvedic system and the Greco-Arabian Unani system of medicine. The World Health Organization (WHO) defines TM as health practices, approaches, knowledge and beliefs incorporating plant-, animaland mineral-based medicines, spiritual therapies, manual techniques and exercises applied singularly or in combination to treat, diagnose and prevent illness or maintain well-being. ${ }^{1,2}$ In this respect, the terms 'complementary' and 'alternative' medicine are used to refer to a wide-ranging set of healthcare practices that are not a part of a country's own tradition or are not integrated into its dominant healthcare system, but a part of other countries' traditional practices. ${ }^{1}$ For example, acupuncture and Indian Ayurvedic practices are part of traditional Chinese and Indian medicine, respectively, but many Western countries consider them as complimentary or alternative practices as they are not part of their own traditional practices. In fact, traditional, complementary and alternative health practices are very common and the WHO estimates that approximately $70-80 \%$ of populations in both developing and developed countries use them either singularly or alongside modern therapies. ${ }^{2}$

The uses and forms of TM practices vary from country to country and, indeed, even between different regions in a particular country. ${ }^{3}$ TM practices constitute the healthcare cultures of many Asian and African countries. ${ }^{3,4}$ Many of these practices, like the Indian Ayurvedic system or the African or Chinese 
systems, are centuries old and have been handed down through generations either by word-of-mouth or via written scripts. Some of these practices are related to the use of herbs or animal/plant products, or they might be related to physical practices such as traditional massages, acupuncture, exercises and others such as cupping and branding., ${ }^{2,4}$ In the past, the practitioners of TM in many developing countries were the only sources of healthcare, especially in remote areas, due to poor access to modern hospitals and doctors that were expensive and, at times, far away. ${ }^{5}$ With improved access to modern healthcare in developing countries, the number of these TM practitioners has dwindled considerably. ${ }^{6}$ Despite this trend, many of the TM practices are embedded in the cultures and customs of many communities, presently coexisting along with modern hospitals and modern medicine. $^{4}$

Notably, there is currently a renewed interest in traditional and complementary healthcare practices in the Western world and even in many developing countries. ${ }^{4,7}$ One reason behind such an interest is that TM is considered to be a more natural form of medicine and, therefore, free from the side-effects of harmful chemicals. Patients also tend to use TM to treat chronic conditions such as diabetes, hypertension or chronic aches and pains, especially back pain. Even a decade ago, a study from Australia suggested that approximately $70 \%$ of its population used at least one form of complementary medication, with an estimated annual expenditure of more than 3 billion USD on traditional and complementary medications in $2007 .{ }^{8}$ More recently, in the USA, around 32 billion USD was spent on nutritional supplements in 2012; this expenditure is expected to increase to 60 billion USD in 2021. ${ }^{7}$ Furthermore, the global market for complimentary medicines is valued at approximately 82 billion USD; this valuation is expected to experience an estimated rise to more than $22 \%$ by $2028 .{ }^{\circ}$

Oman has a rich heritage regarding the use of TM, which has been practiced in the country throughout the past centuries. ${ }^{10}$ Oman has a rich, biodiverse flora and fauna due to its varied terrain comprising mountains, deserts and river beds (wadis), which has led to the development of herbal medications unique to the region. ${ }^{11}$ This development has also been greatly influenced by Oman's historic ties with other civilisations. Indeed, Oman has a long history of trade (for centuries) with countries in Africa, the Indian subcontinent and more distant places such as Europe and China. As a result, some of the TM practices in Oman have derived important aspects from the TM cultures of these civilisations. ${ }^{11,12}$ Some of the common TM practices in Oman include traditional massages with herbal oils, branding (known locally as wassam), cupping (known locally as hijama), herbal medications and, more recently, acupuncture. ${ }^{12}$

With the urbanisation of the Omani population and the improved access to modern healthcare, even in the rural areas of Oman, it is not clear whether patients still seek out TM practices for healthcare purposes. Hence, this study aimed to assess the knowledge, attitudes and practices among the general Omani population with regard to TM practices. In addition, this study sought to identify the factors that affect the utilisation of these practices. The findings of this study can help governmental institutions of Oman devise strategies to control and incorporate TM practices in modern medicine.

\section{Methods}

This cross-sectional, questionnaire-based study was conducted among the general population in Muscat, Oman, between November 2019 and March 2020. All adults above 18 years of age were eligible to take part in the study; those under 18 years of age and not willing to participate were excluded. For the purpose of sampling, a convenience sampling method was used. Thus, the subjects were recruited from malls and public places during health promotion events. In addition, the call for participants was also posted and circulated on social media as a link.

The questionnaire of this study was self-developed and derived from other similar studies. ${ }^{6,8,13-15}$ It consisted of 21 items divided into two sections. The first section comprised the demographic data of the participants and consisted of five questions. The second section (of 16 questions) collected data about the participants' knowledge, attitudes and practices with regard to TM practices. To test their knowledge, each participants was asked five (yes or no) questions about the use of TM, the potential risks of TM, the side-effects of TM and the need for TM training. The respondents' attitudes and practices were measured in relation to 11 (yes or no) questions that focussed on the history of TM use, the promotion of TM methods, the method's efficacy and the desire for re-use.

Importantly, this questionnaire was devised in the Arabic language and was modified, reviewed and adjusted based on interviews with lay members of the public about the various TM practices that are available in Oman. It was piloted on 50 volunteers, with minor concomitant adjustments made to the language to remove ambiguity, improve clarity and render it eligible for use as the final questionnaire. No questions were deleted following the pilot. The final Cronbach's 
alpha was $0.78,0.83$ and 0.79 for the knowledge, attitude and practice questions, respectively. The overall Cronbach's alpha value was 0.81 , demonstrating an acceptable level of reproducibility.

Sample size estimations were made according to the existing recommendations for population-based cross-sectional questionnaire studies, according to which a minimum of 384 to 400 participants are required for a standard error of $5 \% .{ }^{16}$ The data obtained through the questionnaire were analysed using Statistical Package for the Social Sciences (SPSS), Version 21 (IBM Corp., Armonk, New York, USA). All data were described as either percentages or mean \pm standard deviation or median (interquartile range). Moreover, for this study, Student's t-test, Mann-Whiney U test and Chi-squared test were considered as appropriate. Binary logistic regression was used to predict the use of any TM practice individually or in combination. In addition, demographic factors associated with the study participants were used as predictors. A $P$ value $<0.05$ was considered to be significant. Data were stored securely in a password-protected file that was only accessible by the investigators.

Ethical approval for the study was granted by the Medical Research Ethics Committee, College of Medicine and Health Sciences at Sultan Qaboos University (MREC\#2309), prior to its commencement. Prior to answering the questionnaire, the rationale and reason for conducting the study was explained to the participants, after which they had to sign a consent statement on the questionnaire. Those responding to the electronic questionnaire had to tick a box stating that they had read the study rationale, understood it and consented to participation.

\section{Results}

Out of the 700 questionnaires that were distributed, 598 replies were received (response rate: $85.4 \%$ ), of which 46 were deemed incomplete. Therefore, a total of 552 responses were included in the final analysis. The mean age of the respondents was $33.6 \pm 7.7$ years and most were male (62.5\%). There were 32 diabetics (5.8\%), 22 hypertension patients $(4.0 \%)$ and 14 patients with heart disease (2.5\%). The majority of the respondents (88.9\%) did not indicate any cardiovascular risk factors. Additionally, most of the respondents were employed full-time (78.1\%); 3.4\% of the respondents were full-time students. Nearly three quarters of the respondents had completed a university diploma or had a higher educational qualification [Table 1].

Most respondents knew about/were aware of the different types of TM methods: wassam $(n=476$,
Table 1: Characteristics of the questioned urban population in Muscat, Oman $(\mathrm{N}=552)$

$\begin{array}{lc}\text { Characteristic } & \mathbf{n}(\%) \\ \text { Mean age } \pm \text { SD in years } & 33.6 \pm 7.7 \\ \text { Gender } & \\ \text { Male } & 345(62.5) \\ \text { Female } & 207(37.5) \\ \text { Comorbidity* } & \\ \text { Diabetic } & 32(5.8) \\ \text { Hypertensive } & 22(4.0) \\ \text { Heart disease } & 14(2.5) \\ \text { No cardiovascular risk factors } & 491(88.9) \\ \text { Employment status } & \\ \text { Student } & 19(3.4) \\ \text { Employed full-time } & 431(78.1) \\ \text { Unemployed/retired } & 102(18.5) \\ \text { Educational status } & \\ \text { Less than secondary school } & 24(4.3) \\ \text { Completed secondary school } & 118(21.4) \\ \text { Diploma or higher } & 410(74.3) \\ \text { "Some patients had more than one comorbidity. } & \end{array}$

86.2\%), cupping ( $\mathrm{n}=495,89.7 \%)$, traditional massage ( $\mathrm{n}=427,77.4 \%)$, herbal medications ( $\mathrm{n}=461,83.5 \%)$ and acupuncture $(\mathrm{n}=384,69.6 \%)$. Although most felt that TM was not better than modern medicine (80.3\%), many $(81.5 \%)$ felt that it was effective and approximately two-thirds $(\mathrm{n}=374,67.8 \%)$ had tried at least one TM method for healthcare purposes. Most male respondents (78.3\%) had tried some form of TM practice compared to approximately half of the female respondents (50.2\%). Of those who had tried it, a majority $(\mathrm{n}=315,84.2 \%)$ found it to be useful, with only a small number $(\mathrm{n}=31,8.3 \%)$ claiming that they had subsequently suffered some side-effects [Table 2].

Indeed, most of the respondents who had used TM said that they would try it again (85.3\%), although only a few stated that they would recommend its use to others (36.1\%). Among those who had not tried TM, most did not have any specific reason for not trying it (94.4\%), whereas $30.9 \%$ said that they did not know enough about TM to want to try it. Crucially, there was no significant difference regarding the TM-related attitudes and practices between males and females, apart from the fact that there was a significantly higher proportion of males who had tried some form of TM than females $(P<0.001)$; males were also being more likely to try it again $(P<0.001)$ [Table 2]. 
Table 2: Knowledge and attitudes regarding traditional and complementary medicine practices $(\mathrm{N}=552)$

\begin{tabular}{|c|c|c|c|c|}
\hline \multirow{2}{*}{ Variable } & \multicolumn{3}{|c|}{ n (\%)* } & \multirow{2}{*}{$\begin{array}{c}P \\
\text { value }^{\dagger}\end{array}$} \\
\hline & Total & $\begin{array}{c}\text { Male } \\
(\mathrm{n}=345)\end{array}$ & $\begin{array}{l}\text { Female } \\
(\mathrm{n}=207)\end{array}$ & \\
\hline \multicolumn{5}{|l|}{ Are you aware of: } \\
\hline Wassam & $476(86.2)$ & $291(84.3)$ & $185(89.3)$ & 0.09 \\
\hline Cupping & 495 (89.7) & $314(91)$ & $181(87.4)$ & 0.1 \\
\hline Massage & 427 (77.4) & $253(73.3)$ & $174(84.1)$ & 0.04 \\
\hline $\begin{array}{l}\text { Herbal } \\
\text { medications }\end{array}$ & $461(83.5)$ & $286(82.8)$ & $175(84.5)$ & 0.6 \\
\hline Acupuncture & $384(69.6)$ & $232(67.2)$ & $152(73.4)$ & 0.1 \\
\hline $\begin{array}{l}\text { Do you feel } \\
\text { traditional } \\
\text { medicine is } \\
\text { effective? }\end{array}$ & $450(81.5)$ & $291(84.3)$ & $159(76.8)$ & 0.02 \\
\hline $\begin{array}{l}\text { Do you think } \\
\text { it is better } \\
\text { than modern } \\
\text { medicine? }\end{array}$ & 109 (19.7) & $69(20)$ & $40(19.3)$ & 0.8 \\
\hline $\begin{array}{l}\text { Have you ever } \\
\text { tried any? }\end{array}$ & $374(67.8)$ & $270(78.2)$ & $104(50.2)$ & $<0.001$ \\
\hline $\begin{array}{l}\text { Did you try it for } \\
\text { hypertension? }\end{array}$ & $\begin{array}{l}(\mathrm{n}=22) \\
17(77.3)\end{array}$ & $\begin{array}{l}(\mathrm{n}=15) \\
13(86.7)\end{array}$ & $\begin{array}{l}(\mathrm{n}=7) \\
4(57.1)\end{array}$ & 0.1 \\
\hline $\begin{array}{l}\text { Did you try it for } \\
\text { reducing blood } \\
\text { sugar? }\end{array}$ & $\begin{array}{c}(\mathrm{n}=32) \\
18(56.3)\end{array}$ & $\begin{array}{c}(\mathrm{n}=17) \\
10(58.8)\end{array}$ & $\begin{array}{l}(n=15) \\
8(53.3)\end{array}$ & 0.6 \\
\hline $\begin{array}{l}\text { For those who } \\
\text { had tried it: }\end{array}$ & $(\mathrm{n}=374)$ & $(\mathrm{n}=270)$ & $(\mathrm{n}=104)$ & \\
\hline Was it useful? & $315(84.2)$ & $228(84.4)$ & 87 (83.7) & 0.5 \\
\hline $\begin{array}{l}\text { Did you have any } \\
\text { side effects? }\end{array}$ & $31(8.3)$ & $21(7.8)$ & $10(9.6)$ & 0.6 \\
\hline $\begin{array}{l}\text { Will you try it } \\
\text { again in future? }\end{array}$ & 319 (85.3) & $163(78.7)$ & $89(85.6)$ & $<0.001$ \\
\hline $\begin{array}{l}\text { Did you use } \\
\text { it alongside } \\
\text { modern } \\
\text { medications? }\end{array}$ & $186(49.7)$ & $125(60.4)$ & $61(58.7)$ & 0.07 \\
\hline $\begin{array}{l}\text { Would you } \\
\text { recommend its } \\
\text { use? }\end{array}$ & $135(36.1)$ & $103(38.1)$ & $31(29.8)$ & 0.1 \\
\hline $\begin{array}{l}\text { If you have not } \\
\text { used any, why } \\
\text { not? }\end{array}$ & $(\mathrm{n}=178)$ & $(\mathrm{n}=75)$ & $(\mathrm{n}=103)$ & \\
\hline Not effective & $67(37.6)$ & 37 (49.3) & $30(29.1)$ & \\
\hline $\begin{array}{l}\text { Don't know } \\
\text { where available }\end{array}$ & $4(2.2)$ & $4(5.3)$ & $0(0.0)$ & \\
\hline Expensive & $8(4.5)$ & $7(9.3)$ & $1(1.0)$ & \\
\hline $\begin{array}{l}\text { Don't know } \\
\text { enough of it }\end{array}$ & $55(30.9)$ & $32(42.7)$ & $23(22.3)$ & 0.04 \\
\hline $\begin{array}{l}\text { No specific } \\
\text { reason }\end{array}$ & $168(94.4)$ & $70(93.3)$ & $98(95.1)$ & \\
\hline
\end{tabular}

Table 3: Characteristics of respondents who have tried any form of traditional treatment $(\mathrm{N}=552)$

\begin{tabular}{llcl} 
Characteristic & \multicolumn{2}{c}{ n (\%) } & P value* \\
& $\begin{array}{l}\text { Not tried } \\
(\mathbf{n}=\mathbf{1 7 8})\end{array}$ & $\begin{array}{c}\text { Tried } \\
(\mathbf{n}=374)\end{array}$ & \\
& $31.8 \pm 7.2$ & $34.5 \pm 7.8$ & $<0.001^{+}$ \\
$\begin{array}{l}\text { Mean age } \pm \\
\text { SD in years }\end{array}$ & & & \\
Gender & $75(42.1)$ & $270(72.2)$ & \\
Male & $103(57.9)$ & $104(27.8)$ & $<0.001$ \\
Female & &
\end{tabular}

\section{Employment status}

Student $\quad 13(7.3) \quad 6(1.6)$

Employed full- $\quad 116(65.2) \quad 315(84.2)$

time

Unemployed/ $\quad 49(27.5) \quad 53(14.2) \quad<0.001$

retired

Educational status

Primary $\quad 6(3.3) \quad 18(4.8)$

school or less

Secondary $\quad 34(19.1) \quad 84(22.5)$

school

Diploma or $\quad 138(77.5) \quad 272(72.7) \quad 0.4$

higher

Comorbidity

Diabetes

$$
7 \text { (3.9) }
$$

$15(4.0)$

0.9

Hypertension

7 (3.9)

$25(6.7)$

0.19

Heart disease

$3(1.7)$

$11(2.9)$

0.38

"Using Chi-squared test.

+Using students t-test.

Those who had reportedly tried at least one TM practice were older than those who had not (34.5 \pm 7.8 versus $31.8 \pm 7.2$ years; $P<0.001$ ) and more males had tried TM compared to males who had not $(72.2 \%$ versus $42.1 \%$; $P<0.001)$. Most of those who had tried a TM practice were actively employed (84.2\%), with around $15 \%$ in full-time education and/or unemployed or retired. However, there was no difference in the respondents' TM practices with regard to their educational statuses. Similarly, neither did any cardiovascular risk factor influence their use of TM practices [Table 3].

Furthermore, the type of TM practice utilised by respondents did not vary according to age, gender, employment status or educational status. Herbal medications (65.8\%) and traditional massage (60.4\%) were the most common forms of TM reportedly practiced. Females more often chose herbal medications (69.2\%) and massage (63.5\%) rather than cupping (29.8\%), wassam (24.0\%) and/or acupuncture 
Table 4: Characteristics of those trying each different modality of traditional practice $(n=374)$

\begin{tabular}{|c|c|c|c|c|c|}
\hline \multirow[t]{2}{*}{ Characteristic } & \multicolumn{5}{|c|}{ n (\%) } \\
\hline & $\begin{array}{l}\text { Tried wassam } \\
\quad(\mathbf{n}=151)\end{array}$ & $\begin{array}{l}\text { Tried cupping } \\
\quad(\mathbf{n}=207)\end{array}$ & $\begin{array}{l}\text { Tried massage } \\
\qquad(\mathrm{n}=226)\end{array}$ & $\begin{array}{l}\text { Tried herbal } \\
\text { medication } \\
(\mathbf{n}=246)\end{array}$ & $\begin{array}{c}\text { Tried } \\
\underset{(\mathbf{n}=71)}{\text { acupuncture }}\end{array}$ \\
\hline Mean age $\pm S D$ in years & $34.6 \pm 7.8$ & $34.8 \pm 7.5$ & $34.2 \pm 8.2$ & $34.9 \pm 8.5$ & $35.9 \pm 9.4$ \\
\hline \multicolumn{6}{|l|}{ Gender } \\
\hline Male & $126(83.4)$ & $176(85.0)$ & $160(70.8)$ & $174(70.7)$ & $59(83.1)$ \\
\hline Female & $25(16.6)$ & $31(15.0)$ & $66(29.2)$ & $72(29.3)$ & $12(16.9)$ \\
\hline \multicolumn{6}{|l|}{ Employment status } \\
\hline Student & $2(1.3)$ & $2(1.0)$ & $3(1.3)$ & $3(1.2)$ & $0(0.0)$ \\
\hline Employed full-time & $135(89.4)$ & $180(87.0)$ & $192(85.0)$ & 199 (80.9) & $62(87.3)$ \\
\hline Unemployed/retired & $14(9.3)$ & $25(12.1)$ & $31(13.7)$ & $44(17.9)$ & $9(12.7)$ \\
\hline \multicolumn{6}{|l|}{ Educational status } \\
\hline Primary school or less & $6(4.0)$ & $13(6.3)$ & $10(4.4)$ & $16(6.5)$ & $5(7.0)$ \\
\hline Secondary school & $40(26.5)$ & $53(25.6)$ & $62(27.4)$ & $50(20.3)$ & $20(28.2)$ \\
\hline Diploma or higher & $105(69.5)$ & $141(68.1)$ & $154(68.1)$ & $180(73.1)$ & $46(64.8)$ \\
\hline \multicolumn{6}{|l|}{ Comorbidity } \\
\hline Diabetic & $7(4.6)$ & $7(3.4)$ & $6(2.7)$ & $10(4.1)$ & $3(4.2)$ \\
\hline Hypertensive & $7(4.6)$ & $17(8.2)$ & $14(6.2)$ & $18(7.3)$ & $6(8.5)$ \\
\hline Heart disease & $7(4.6)$ & $7(3.4)$ & $5(2.2)$ & $7(2.8)$ & $3(4.2)$ \\
\hline
\end{tabular}

Table 5: Distribution of the traditional practices with the symptoms $(\mathrm{n}=374)$

$\begin{array}{lccccc}\text { Symptom } & \begin{array}{c}\text { Wassam } \\ (\mathbf{n}=\mathbf{1 5 1})\end{array} & \begin{array}{c}\text { Cupping } \\ (\mathbf{n}=\mathbf{2 0 7})\end{array} & \begin{array}{c}\text { Traditional } \\ \text { massage } \\ (\mathbf{n}=\mathbf{2 2 6})\end{array} & \begin{array}{c}\text { Herbal } \\ \text { medications } \\ (\mathbf{n}=\mathbf{2 4 6})\end{array} & \begin{array}{c}\text { Acupuncture } \\ (\mathbf{n}=\mathbf{7 1})\end{array} \\ \text { Headache }(\mathrm{n}=110) & 44 & 77 & 69 & 73 & 28 \\ \text { Back pain }(\mathrm{n}=202) & 75 & 140 & 150 & 126 & 43 \\ \text { Abdominal pain }(\mathrm{n}=126) & 55 & 43 & 67 & 100 & 10 \\ \text { Nerve pain }(\mathrm{n}=147) & 57 & 97 & 106 & 103 & 31 \\ \text { Jaundice }(\mathrm{n}=62) & 48 & 34 & 25 & 31 & 7 \\ \text { Swelling }(\mathrm{n}=36) & 19 & 18 & 22 & 29 & 4\end{array}$

(11.5\%). Among the male respondents, cupping was the most common form of TM (65.2\%) followed by herbal medications (64.4\%), massage $(59.3 \%)$, wassam (46.7\%) and acupuncture (21.9\%). Of the diabetic and hypertensive patients, $66.7 \%$ and $72.0 \%$, respectively, had reportedly tried herbal medications, with many claiming that they had specifically used it to lower blood sugar (56.2\%) or blood pressure (77.2\%) [Table 4].

Back pain was the most common condition for which TM was used, with the majority having tried traditional massage (74.3\%), cupping (69.3\%) and/or herbal medications (62.4\%) to treat it. Acupuncture was found to be the least common TM practice (21.2\%) used for back pain. The other conditions treated using TM were headache, abdominal pain, 'nerve' pain, jaundice and swelling [Table 5].

A binary logistic regression revealed that age $(P<0.001)$ and gender $(P<0.001)$ strongly predicted the use of any TM practice. Other factors such as educational qualification or employment status were not predictive in this regard. However, gender specifically predicted the use of wassam, cupping and acupuncture $(P<0.001$ each). Knowledge regarding a specific practice also predicted the uses of wassam $(P$ $=0.04)$, cupping $(P<0.001)$, massage $(P<0.001)$ and 
herbal medications $(P<0.001)$. Employment status only predicted the use of herbal medication $(P=0.01)$.

\section{Discussion}

In Oman, there appears to be a recurrent and widespread use of TM practices for a range of ailments. According to this study, the proportion of people who reportedly used some form of TM was $67.8 \%$, similar to proportions reported in other studies from countries such as Ethiopia, Nigeria, Ghana, India, China and South Africa. ${ }^{6,13-15,17,18}$ As in other countries, TM is usually used in Oman to mainly treat chronic conditions that did not respond to modern medications or for ailments such as diabetes, low-back pain, muscular pain, etc. Many TM users asserted that they tolerated it well, found it useful and would recommend its use. The patterns of TM use, however, suggested that the more physical forms of TM practices such as massage, acupuncture, wassam and cupping were used more by males, while females tended to use herbal medications more than other forms. This finding was consistent with those from studies across different cultures. ${ }^{6,13-15,17,18}$

Consistent with other studies, herbal therapy was the most common form of TM that was utilised among the current respondents. ${ }^{15,19}$ Indeed, herbal medications can be used for a wide variety of ailments and are considered to be safe and generally free from side-effects, as they are derived from naturally occurring plants and herbs. Traditional herbal therapy is common in many cultures, usually with the indigenous herbs and plants that are unique to a particular country or region. In fact, studies from the Middle East demonstrate a high level of herbal medications' use for various ailments and especially during pregnancy. ${ }^{20,21}$ Concerning Oman, many of the traditional Omani herbal medications contain rose water, lime and local honey which are plentiful in the hilly ranges of Jebal Akhdar. ${ }^{11,12}$ Moreover, the leaves, resin, bark and sap of plants found in these hills are also used extensively to prepare herbal medications. ${ }^{11,12}$ In addition, the Dhofar region of Oman is famous for frankincense, which has been used for centuries as incense as well as for its therapeutic properties. ${ }^{22}$ To be specific, frankincense forms a part of many Omani herbal medications, as a paste, an inhalant, an ointment or even a product to be ingested. In view of this abundance of local medicinal herbs and plants, it is not surprising that herbal medications were considered the most common form of TM in the current study. The use of herbal medications is widespread and generally unregulated in most countries; although they appear to be tolerated well by the current respondents, a note of caution is necessary: patients may develop unexpected side-effects especially with prolonged or excessive TM use. Therefore, this study recommends the inclusion of patients' histories of herbal medications' ingestion as part of routine check-ups. ${ }^{21}$

Traditional massage therapy is common all over the world and is used to treat a variety of conditions. It evidently decreases muscular and joint pain, labour pain, improves mood, reduces anxiety, improves quality of sleep and reduces blood pressure in the short term, although data on its long-term benefits are inadequate. ${ }^{23,24}$ However, it is readily available, presumed safe and often has an immediate effect on pain and mood, which is most likely the reason for its popularity. ${ }^{25}$ There are various ways of explaining the effects of massage on pain and mood. For instance, the pressure and local heat caused by rubbing and massaging improves blood flow and local vasodilation. There are also massage-related neurohormonal changes such as increased dopamine and decreased noradrenaline levels, along with changes in parasympathetic activity and neuronal excitability. ${ }^{26}$ Thus, among the respondents of this study, massage emerged as the second-most common form of TM practice. However, like the other forms of TM, it appeared to be predominantly used by males and employed individuals. For females, massage was the second-most common form of TM and was used for most indications of ailments.

Branding is a common practice in many countries. ${ }^{27}$ It involves applying a hot metal object to the affected part of the body; the subsequent thirddegree burns are considered to be a counter-irritant to the original ailment. Although branding is dangerous and potentially harmful, it is widely practiced in many countries and known by different names, such as wassam in Oman, guboow in Somalia, kaiy in Libya, etc. ${ }^{27,28}$ Despite its popularity, there are many case reports of complications related to branding. ${ }^{12,28}$ In the current study's cohort, it was not the most commonly used practice and was utilised mainly by older men. Almost half of the males who had practiced some form of TM said that they had used branding, suggesting that it is still popular despite the associated pain and potential harm.

Cupping therapy is another common physical TM method used in many parts of Asia and the Middle East, with a growing popularity in Western countries. There are two types of cupping: wet cupping (known as hijama in Oman) and dry cupping. This practice involves the application of a bamboo, glass or plastic cup to the area of treatment. A vacuum is then created either manually or via electromagnetic suction inside the cup in order to draw the skin into it. In wet cupping, 
blood is drawn into the cup via a small incision made prior to its application, while in dry cupping no blood is drawn. The mechanism of action associated with cupping is not clear, and many theories have been proposed in this respect. Some suggest that it acts by triggering a diffuse noxious inhibitory control or by the removal of oxidants and the decrease of oxidative stress at a local level. Others hypothesise that this therapy does the following: (1) drains excess fluids; (2) increases blood flow to skin and muscles; and (3) stimulates the peripheral nervous, neurohormone, circulatory and immune systems. ${ }^{29}$ There are numerous clinical trials and meta-analyses of its use in a variety of conditions such as back pain, neck pain, migraine, hypertension and chronic obstructive pulmonary disease. ${ }^{29-31}$ The results of these studies are variable, with some studies showing benefits compared to placebo or standard therapy whilst others do not. Notably, interest in cupping has increased after some celebrity athletes have reportedly used it. ${ }^{32}$ In this study's cohort, less than half of those who had used some form of TM revealed the use of cupping, similar to the observations regarding wassam. This demonstrated that there is still a considerable interest in cupping as a form of TM therapy.

Acupuncture was the least common of the TM practices utilised in the current study. It is a Chinese TM practice that involves the placing of needles in special locations which can affect the pain sensations in the body. It is suggested that by affecting afferent nerve signalling, acupuncture can lead to the release of endogenous opioids and thereby reduce pain. ${ }^{33}$ Although it has been a part of Chinese traditional practice for many centuries, it is only within the last few decades that acupuncture has gained popularity and acceptance in much of the Western world. ${ }^{34}$ However, inconsistent clinical results, limited availability (as it has to be provided by specially trained professionals) and misconceptions about its use (such as pain and other complications) limit its availability and overall appeal compared to other forms of TM. ${ }^{35}$ In the current study, other 'painful' and potentially harmful practices such as wassam were found to be more popular than acupuncture. This could be due to the respondents' familiarity with wassam, as it has been practiced in the studied region for a long time.

This study found no clearly defined demographic factors that could predict the use of TM. As in other studies from the region, massage therapy and herbal medications were used by both males and females, while males additionally used the more 'painful' physical therapies such as wassam, cupping and acupuncture. ${ }^{19}$ Moreover, TM was used by a high proportion of those that were employed compared to students and those who were unemployed/retired; this finding perhaps reflects the cost-related implications of using TM.

One of the limitations of this study was that it was confined to the population living in the urban areas of Oman's capital city, Muscat. The TM use and related views of the population living in the rural areas of Oman could be different. In addition, the present study's cohort had a high proportion of young, educated and employed individuals, suggesting their relatively higher socioeconomic status (although data on household income was not collected). Thus, the concomitant views and practices of older people and those with no formal education beyond primary schooling could be different and were not represented in the current study. Similarly, the practices and attitudes towards TM in the rural areas of Oman could be different. Despite these limitations, the sample size of this study was large and provided insights into the knowledge, attitudes and practices relating to TM in modern-day Oman. Additionally, this was the first study to assess the attitudes of the Omani population regarding TM practices.

This study demonstrates the widespread use of TM practices among the urban population in Oman. The use of these practices is deeply embedded in the social culture of the Omani population, with many availing of their benefits. In many countries, efforts have been undertaken to try and integrate these practices to complement modern healthcare practices. ${ }^{2,3,5,6}$ Although this study provides valuable insight into such practices and the related attitudes, more research is required to improve the understanding of Oman's local TM practices, so that they can be fully integrated into the local healthcare strategies. Undoubtedly, many patients seem to benefit from these traditional practices and utilising them to complement modern healthcare can immensely improve the overall health of Oman's population.

\section{Conclusion}

Despite the availability and ease of access to modern healthcare, there is still widespread use of traditional medicinal practices alongside modern medicine in urban Oman. Traditional practices are part of the culture and heritage of various communities, though these practices appear to be used less frequently by Oman's youth compared to its older population. In this light, interventions to educate Oman's public regarding the side-effects of some of the more harmful methods of TM are imperative. At the same time, a better understanding of the mechanisms underlying the benefits of some of these practices is needed to 
aid their improved integration into Oman's local healthcare services.

\section{AUTHORS' CONTRIBUTION}

HAR and AAM collected the data. AAM, SKN and MA contributed to the writing of the manuscript. SKN analysed the data. All authors approved the final version of the manuscript.

\section{CONFLICT OF INTEREST}

The authors declare no conflicts of interest.

\section{FUNDING}

No funding was received for this study.

\section{References}

1. WorldHealthOrganization.Traditionalmedicine:Definitions.From: https://www.who.int/traditional-complementary-integrative -medicine/en/ Accessed: Nov 2021.

2. World Health Organization. WHO: Traditional medicine strategy 2014-2023. From: https://apps.who.int/iris/handle/10665/92455 Accessesd: Nov 2021

3. Oyebode O, Kandala NB, Chilton PJ, Lilford RJ. Use of traditional medicine in middle-income countries: A WHO-SAGE study Health Policy Plan 2016; 31:984-91. https://doi.org/10.1093/ heapol/czw022.

4. World Health Organization. WHO global report on traditional complimentary and integrative medicine 2019. From: https:// www.who.int/traditional-complementary-integrative-medicine/ WhoGlobalReportOnTraditionalAndComplementaryMedicine 2019.pdf Accessed: Nov 2021.

5. Birhan W, Giday M, Teklehaymanot T. The contribution of traditional healers' clinics to public health care system in Addis Ababa, Ethiopia: A cross-sectional study. J Ethnobiol Ethnomed 2011; 7:39. https://doi.org/10.1186/1746-4269-7-39.

6. Angmo K, Adhikari BS, Rawat GS. Changing aspects of traditional healthcare system in western Ladakh, India. J Ethnopharmacol 2012; 143:621-30. https://doi.org/10.1016/j.jep.2012.07.017.

7. Nutritional Supplements Flexing Muscles As Growth Industry From: https://www.forbes.com/sites/davidlariviere/2013/04/18/ nutritional-supplements-flexing-their-muscles-as-growthindustry/?sh=2d57956b8845 Accessed: Nov 2021.

8. Xue CC, Zhang AL, Lin V, Da Costa C, Story DF. Complementary and alternative medicine use in Australia: A national populationbased survey. J Altern Complement Med 2007; 13:643-50. https:// doi.org/10.1089/acm.2006.6355.

9. Complementary And Alternative Medicine Market Size, Share \& Trends Analysis Report By Intervention (Botanicals, Mind Healing, Body Healing, External Energy, Sensory Healing), By Distribution Method, And Segment Forecasts, 2021 - 2028. From: https://www.grandviewresearch.com/industry-analysis/ complementary-alternative-medicine-market Accessed: Nov 2021.

10. Azaizeh H, Saad B, Cooper E, Said O. Traditional Arabic and Islamic medicine, a re-emerging health aid. Evid Based Complement Alternat Med 2010; 7:419-24. https://doi.org/10.1093/ecam/ nen039.

11. Divakar MC, Al-Siyabi A, Varghese SS, Rubaie MA. The practice of ethnomedicine in the northern and southern provinces of Oman. Oman Med J 2016; 31:245-52. https://doi.org/10.5001/ omj.2016.49.
12. Shenoy R, Bialasiewicz A, Khandekar R, Al Barwani B, Al Belushi $\mathrm{H}$. Traditional medicine in Oman: Its role in ophthalmology. Middle East Afr J Ophthalmol 2009; 16:92-6. https://doi. org/10.4103/0974-9233.53869.

13. Wassie SM, Aragie LL, Taye BW, Mekonnen LB. Knowledge, attitude, and utilization of traditional medicine among the communities of Merawi Town, Northwest Ethiopia: A crosssectional study. Evid Based Complement Alternat Med 2015; 2015:138073. https://doi.org/10.1155/2015/138073

14. Aina O, Gautam L, Simkhada P, Hall S. Prevalence, determinants and knowledge about herbal medicine and non-hospital utilisation in southwest Nigeria: A cross-sectional study. BMJ Open 2020; 10:e040769. https://doi.org/10.1136/bmjopen-2020-040769.

15. Sato A. Revealing the popularity of traditional medicine in light of multiple recourses and outcome measurements from a user's perspective in Ghana. Health Policy Plan 2012; 27:625-37. https:// doi.org/10.1093/heapol/czs010.

16. Sample size calculations for questionairre based studies. From: https://issuu.com/medecinsdumonde/docs/47-the-kap-surveymodel-knowledge-a/9 Accessed: Nov 2021.

17. Xin B, Mu S, Tan T, Yeung A, Gu D, Feng Q. Belief in and use of traditional Chinese medicine in Shanghai older adults: A cross-sectional study. BMC Complement Med Ther 2020; 20:128. https://doi.org/10.1186/s12906-020-02910-x.

18. Nxumalo N, Alaba O, Harris B, Chersich M, Goudge J. Utilization of traditional healers in South Africa and costs to patients: Findings from a national household survey. J Public Health Policy 2011; 32:S124-36. https://doi.org/10.1057/jphp.2011.26.

19. AlBedah AM. Use of complementary and alternative medicine by cancer patients in Saudi Arabia: A paradox in healthcare. J Altern Complement Med 2013; 19:918-19. https://doi.org/10.1089/ acm.2011.0266.

20. Aljofan M, Alkhamaiseh S. Prevalence and factors influencing use of herbal medicines during pregnancy in Hail, Saudi Arabia: A cross-sectional study. Sultan Qaboos Univ Med J 2020; 20:e71-6. https://doi.org/10.18295/squmj.2020.20.01.010

21. Alkhamaiseh SI, Aljofan M. Prevalence of use and reported side effects of herbal medicine among adults in Saudi Arabia. Complement Ther Med 2020; 48:102255. https://doi.org/10.1016/j. ctim.2019.102255.

22. Al-Yasiry AR, Kiczorowska B. Frankincense--therapeutic properties. Postepy Hig Med Dosw (Online) 2016; 70:380-91. https://doi.org/10.5604/17322693.1200553.

23. Boyd C, Crawford C, Paat CF, Price A, Xenakis L, Zhang W. The impact of massage therapy on function in pain populations-A systematic review and meta-analysis of randomized controlled trials: Part III, surgical pain populations. Pain Med 2016; 17:175772. https://doi.org/10.1093/pm/pnw101.

24. Krinock M, Goyal D, Goel H, Nadar SK. Wanted: Long term studies on massage therapy in hypertension. J Hum Hypertens 2020; 34:741-4. https://doi.org/10.1038/s41371-020-00399-y.

25. Perlman A, Fogerite SG, Glass O, Bechard E, Ali A, Njike VY, et al. Efficacy and safety of massage for osteoarthritis of the knee: A randomized clinical trial. J Gen Intern Med 2019; 34:379-86. https://doi.org/10.1007/s11606-018-4763-5.

26. Nelson NL. Massage therapy: Understanding the mechanisms of action on blood pressure. A scoping review. J Am Soc Hypertens 2015; 9:785-93. https://doi.org/10.1016/j.jash.2015.07.009.

27. Ghazanfar SA. Wasm: A traditional method of healing by cauterisation. J Ethnopharmacol 1995; 47:125-8. https://doi. org/10.1016/0378-8741(95)01270-n.

28. Raza S, Mahmood K, Hakeem A, Polsky S, Haemel A, Rai S, et al. Adverse clinical sequelae after skin branding: A case series. J Med Case Rep 2009; 3:25. https://doi.org/10.1186/1752-1947-3-25. 
29. Kaki A, Sawsan R, Samiha M, Al Jaouni S, Elalah MA, Ibrahim $\mathrm{N}$. Wet cupping reduces pain and improves health-related quality of life among patients with migraine: A prospective observational study. Oman Med J 2019; 34:105-9. https://doi.org/10.5001/ omj.2019.21.

30. Kim S, Lee SH, Kim MR, Kim EJ, Hwang DS, Lee J, et al. Is cupping therapy effective in patients with neck pain? A systematic review and meta-analysis. BMJ Open 2018; 8:e21070. https://doi. org/10.1136/bmjopen-2017-021070

31. Moura CC, Chaves ÉCL, Cardoso ACLR, Nogueira DA, Corrêa HP, Chianca TCM. Cupping therapy and chronic back pain: Systematic review and meta-analysis. Rev Lat Am Enfermagem 2018; 26:e3094. https://doi.org/10.1590/1518-8345.2888.3094.
32. Interest in cupping therapy spikes after Michael Phelps gold win. From: https://www.theguardian.com/sport/2016/aug/08/ cupping-therapy-interest-spikes-michael-phelps-rio-olympics Accessed: Nov 2021.

33. Vanderploeg K, Yi X. Acupuncture in modern society. J Acupunct Meridian Stud 2009; 2:26-33. https://doi.org/10.1016/S20052901(09)60012-1.

34. Hao JJ, Mittelman M. Acupuncture: Past, present, and future. Glob Adv Health Med 2014; 3:6-8. https://doi.org/10.7453/ gahmj.2014.042.

35. Deng S, Zhao X, DU R, He SI, Wen Y, Huang L, et al. Is acupuncture no more than a placebo? Extensive discussion required about possible bias. Exp Ther Med 2015; 10:1247-52. https://doi. org/10.3892/etm.2015.2653. 